\title{
DETECTING BIRDS AND ESTIMATING THEIR VELOCITY VECTORS BY MEANS OF MRL-5 METEOROLOGICAL RADAR
}

\section{Leonid Dinevich, Yosi Leshem, Mark Pinsky and Alexander Sterkin}

\begin{abstract}
Dinevich L., Leshem Y., Pinsky M., Sterkin A. 2004. Detecting birds and estimating their velocity vectors by means of MRL-5 meteorological radar. Ring 26, 2: 35-53.

The computerised bird monitoring system devised on the basis of MRL-5 meteorological radar enables to perform around-the-clock automated bird monitoring. The paper presents an algorithm for identifying bird signals against a background of other objects. The radar signal reflected from a flying bird changes its location in space. In case the flight direction remains unchanged, the coordinates of the corresponding radio echoes obtained as a result of successive azimuth scans form a straight line. This enables to isolate bird radio echoes from those of motionless or chaotically moving objects, such as ground clutter, clouds, precipitation, atmospheric inhomogeneities, etc. In the proposed algorithm, the coordinates thus obtained are used for estimation of vectors of individual birds and bird groups.

In order to identify a bird signal, its fluctuation pattern is analysed. A fluctuation pattern is determined by the frequency of wing-flapping that is characteristic of a certain bird species. The analysis of the spectra of signals obtained in the study indicates that the proposed algorithm enables to isolate bird radio echoes with approximately $85 \%$ confidence. The technique implying simultaneous measurements of signal fluctuation pattern and of the effective scattering area (ESA) forms a promising basis for further studies aimed at determining the species of flying birds by means of radar.
\end{abstract}

L. Dinevich, Y. Leshem, George S. Wise Faculty of Natural Sciences, Dept. of Zoology, TelAviv University, Ramat Aviv, 69978, Israel; M. Pinsky, Institute of the Earth Sciences, Hebrew University of Jerusalem, Givat Ram, Jerusalem, 91904, Israel; A. Sterkin, Brain Research Institute, Weizmann Institute, Rehovot, Israel

Key words: MRL-5 meteorological radar, bird monitoring, bird migration

\section{THE BACKGROUND AND STATEMENT OF THE PROBLEM}

Increasingly high concentrations of aircraft over relatively small areas, as well as striving to achieve maximum speed on light-weight aircraft have brought about certain conflicts between the technological advancement and natural environment. 
Collisions between aircraft and birds in the air appear to be the most significant challenge of this kind (Ganja et al. 1991, Leshem 1999) that may lead to severe crashes resulting in injuring and killing both humans and birds. It is, therefore, an issue of utmost necessity to develop efficient means for estimation and control of ornithological situation as part of ensuring flight safety.

Radars used for bird monitoring enable to perform mapping of bird migration. Initially, aviation radars were used for this purpose, combining the task of monitoring aircraft and observing birds (Eastwood 1967). However, due to their wide directivity pattern, aviation radars proved to be not suitable for determining the height of bird flights (Alfiya 1995).

Currently, meteorological radars have been used for ornithological observations (Bellrose 1967, Gauthreaux 1970). For example, Doppler WSR-88D/98D radar is able to detect birds both in fine and rainy weather conditions (Gauthreaux et al. 1998a, Zrnic and Ryzhkov 1998), which makes it possible to establish the correlation between the value of radar reflectivity and the intensity of bird migration.

As has been shown experimentally (Dinevich and Kaplan 2000, Dinevich et al. 2000), meteorological radars of MRL-5 type are highly efficient and can serve as basis for developing other technical means for bird monitoring and issuing warnings for aviation in case of massive bird migration. Unlike airdrome radars, MRL-5 employs two simultaneously operating high-power transmitters and receivers adjusted at two wavelengths $(3 \mathrm{~cm}$ and $10 \mathrm{~cm})$, as well as an antenna with two symmetrical narrow directivity patterns. The latter makes it possible to determine target coordinates, including the height, with high confidence.

Monitoring bird migration employs radars operating at various wave-length values. According to Buurma and Bruderer (1990) and Buurma (1999), a radar with $5-\mathrm{cm}$ wave length is an ideal tool for bird observations, since using higher wavelengths lessens the probability of detecting small-size targets, while shorter waves increase the capability of detecting smaller targets like insects, which in turn poses a task of isolating bird reflections from those of insects.

In a number of studies (Richardson et al. 1958, Houghton 1964, Rinehart 1966, Chernikov 1979) the value of $10 \mathrm{~cm}$ was suggested as the optimum wavelength for bird monitoring. This finding was proved by Dinevich et al. (2001) who studied the efficiency of bird detection at two wavelengths $(3 \mathrm{~cm}$ and $10 \mathrm{~cm})$ using MRL-5 radar.

The efficiency of employing radars for bird monitoring depends not only on the choice of the optimum wavelength, but also on the level of the radar power, as well as on the availability of Doppler equipment, polarimeters and technical means for signal processing. Depending on the radar design, different characteristics of signal are used for detecting birds and determining their location and flight speed, among them the radar reflectivity (Gauthreaux et al. 1998b), differential polarisation (Dinevich et al. 1990, 1994), spectral characteristics (Chernikov 1979) and signal distribution over space. Those characteristics form the basis for both computerised and manual interactive radar system for bird monitoring (Dinevich et al. 1990). 
The paper presents a method and an algorithm for extracting bird radio echoes against the background of objects like ground clutter, clouds, precipitation, aircraft and atmospheric inhomogeneities. In addition, the proposed approach enables to determine both speed and direction of an individual bird or a group of birds. The study employed MRL-5 radar not equipped with Doppler indicator and did not utilise the polarimeter.

\section{THE MRL-5 RADAR FOR BIRD MONITORING}

\section{Parameters of the radar}

MRL-5 is two-wavelength high-grade meteorological radar designed mainly for determining structure, dynamics and microphysical characteristics of clouds (Abshayev et al. 1980, Abshayev et al. 1984). The radar has a capability of full azimuth scanning $\left(0-360^{\circ}\right)$ and the elevation range of -2 to $90^{\circ}$ in the upper hemisphere. The main parameters of the radar are presented in Table 1.

Table 1

Main parameters of the MRL-5 radar

\begin{tabular}{|c|c|c|c|}
\hline \multirow{2}{*}{ Parameter } & \multirow{2}{*}{ Unit } & \multicolumn{2}{|c|}{ Channel } \\
\hline & & 1 & 2 \\
\hline Carrier frequency & Mhz & 9595 & 2950 \\
\hline Wave length & $\mathrm{cm}$ & 3.14 & 10.15 \\
\hline Peak power & $\mathrm{kW}$ & 250 & 800 \\
\hline Pulse length & $\mu \mathrm{s}$ & $1 / 2$ & $1 / 2$ \\
\hline Repetition frequency & $\mathrm{Hz}$ & $500 / 250$ & $500 / 250$ \\
\hline Diameter of antenna paraboloid & $\mathrm{m}$ & 1.4 & 4.5 \\
\hline Beam width & Deg & 0.5 & 1.5 \\
\hline Gain & $\mathrm{dB}$ & 40 & 39 \\
\hline Azimuth angle rate & rotations/min & $0 \ldots 6$ & $0 \ldots 6$ \\
\hline Elevation angle rate & scans/min & $0 \ldots 6$ & $0 \ldots 6$ \\
\hline Receiver sensitivity & $\mathrm{dB} / \mathrm{W}$ & 130 & 140 \\
\hline
\end{tabular}

The four-year experiment carried out in Israel (Dinevich et al. 2000) has proved that MRL-5 radar can be efficient for obtaining data on height, speed and directions of bird flights. Such data is the basis for issuing warnings for aviation in any weather conditions around the clock. It is important to emphasise that radars of this type are especially suitable for network bird monitoring covering the vast area over which most of spring and autumn intercontinental bird migration takes place. This area includes the entire Central and Eastern Europe, partially the western part of Asia, as well as Africa (Leshem 1999). Many countries in the area, among them: Russia, Ukraine, Moldova, Georgia, Armenia, Bulgaria, Romania, Hungary, the republics of the former Yugoslavia, Finland, Poland and Israel use MRL-5 radars both for monitoring cloud processes and supplying meteorological services for air- 
ports. Provided minor modifications, MRL-5 radars can be efficiently used for monitoring bird migration.

Another important advantage of MRL-5 radar is the ability to carry out observations on two wavelengths $(3.2 \mathrm{~cm}$ and $10 \mathrm{~cm})$. In certain cases, when the targets are large-size birds whose signals considerably exceed the noise level of the receiver, the $3.2 \mathrm{~cm}$ wavelength proves far more efficient. At this wavelength, the directivity pattern is narrower than that at $10 \mathrm{~cm}$, thus the radar signal is less spread and corresponds to the actual target location with maximum accuracy. Radio echo of other ground clutter (hills, buildings, etc.) is better localised, which enables the radar to efficiently detect groups of birds flying in close proximity to ground clutter. By employing the two simultaneously operating transmitters at the two wavelengths, MRL-5 enables to detect the weakest signals from small birds within the $10 \mathrm{~cm}$ range and, at the same time, to quite accurately determine the parameters of target's location (including the height) within the $3 \mathrm{~cm}$ range. It is noteworthy that signals reflected from insects at the $10 \mathrm{~cm}$ wavelength are usually much weaker than bird signals (Glover et al. 1966, Hajovsky et al. 1966). This fact allows to exclude the weak signals from insects in most cases via the signal restriction procedure.

\section{Measurement procedures and the system of signal processing}

The analysis of bird signals is based on the computerised system of cloud data processing (Abshayev et al. 1984), the data being subjected to restriction and filtration. In order to increase the "signal/noise" ratio, signals obtained over several probing impulses are summed up (usually over 16 impulses). The signals are calibrated using the calculated values of the radar constant, the receiver noise being periodically measured. This additional information, alongside with other data, is used to extract bird signals against the background of other objects.

For better efficiency, the data collection is carried out more frequently than it is required by the radar resolution. Each bird is represented in "distance-azimuth" coordinates, the representation looking like a spot rather than a point. The radar's resolution and the recording system parameters are presented in Table 2.

Table 2

Resolutions of the radar and registration units

\begin{tabular}{|l|c|c|}
\hline & Radar & Registration \\
\hline Azimuth resolution & $0.5^{\circ}$ & $0.176^{\circ}$ \\
\hline Distance resolution & $150 \mathrm{~m}$ & $60 \mathrm{~m}$ \\
\hline
\end{tabular}

In order to obtain information on the number and coordinates of birds, azimuth scans are carried out at elevations varying from $0^{\circ}$ to $7-15^{\circ}$. The radar antenna is lifted automatically with a step equal to the width of the corresponding directional pattern, i.e. at each $0.5^{\circ}$ at $3.2 \mathrm{~cm}$ wavelength and at each $1.5^{\circ}$ at $10 \mathrm{~cm}$ wavelength. 
In addition, several scans (typically, 7-15) are performed at the same fixed elevation value. Superposition of data obtained from several scans enables to visually observe the tracks of bird signals.

Figure 1 presents the map of tracks formed by the shifts of bird radio echoes on 21 March 2001, at 9.00 p.m. Superposition of nine azimuth scans was performed at the same fixed elevation value and the rate of 4 scans per minute. In the figure, one can see distinct signal streaks resulting from a two-minute bird motion. Each of these streaks consists of radio echo points consecutively shifting along the direction of the bird's flight. When there is a change in the flight height, the bird goes beyond the antenna directional pattern, and the number of radio echo points forming the corresponding track in this case may fall below the number of the antenna rotations. The length of a track streak is proportional to the average directed velocity of the bird's flight. As can be seen from Figure 1, in cases of a sparse bird group the streaks formed by radio echoes are located at some distance from one another and can be easily identified. It can be seen more distinctly within the square marked $A$ in the figure.

However, in case of a dense mass of birds, their flying tracks intersect, as can be seen in several fragments of the figure, and the streaks formed by radio echo points are difficult to identify. Besides, the radar screen is crowded with signals covering

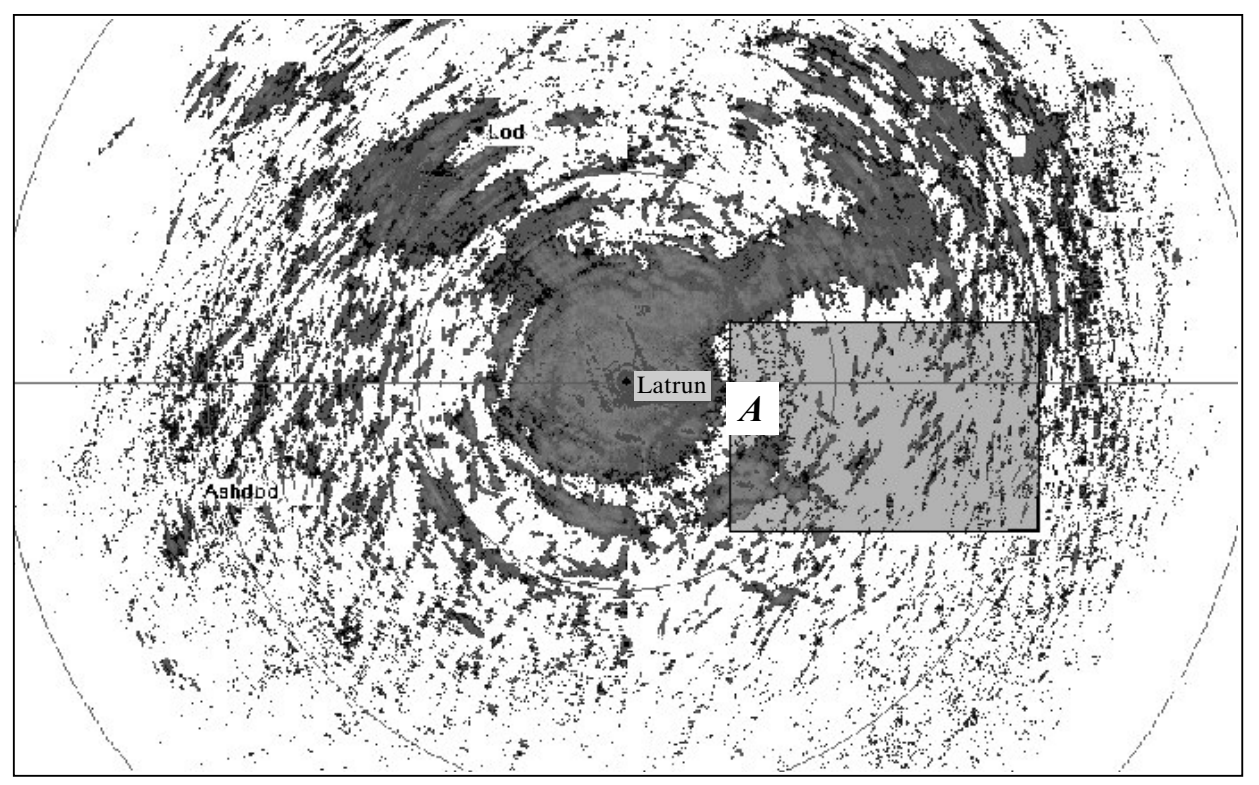

Fig. 1. Chart of bird radio-echo movement. The data were obtained on 21 March 2001, night-time, at wavelength of $10 \mathrm{~cm}$. The location of the radar is designated in the centre of the chart. The difference between circle radiuses is $10 \mathrm{~km}$. Streaks formed by firm lines and lines formed by dot sequences represent radio-echo of moving birds. Areal radio-echo represents reflections from ground clutters. Arc-shaped radio-echo are ground clutter reflections produced by radar side lobes. The zone contained within the framed $A$ rectangular was used as target for subsequent analysis. 
significant areas of ground clutter, obtained from the radar side lobs and shaped as narrow arcs.

Figure 2 shows another sample of superposition, this time of six consecutive azimuth surveys performed at increasing elevation. The data were obtained at daytime on 10 September 2002, at the wavelength of $10 \mathrm{~cm}$, while observing the migration of a large bird flock (Honey buzzard, Pernis apivorus). Summing-up the radio echoes at different heights enables to follow-up large bird flocks over the distance of $100 \mathrm{~km}$.

The band formed by bird radio-echoes had a number of characteristic features. A distinct dynamics of radar signals was observed during the monitoring session. The fluctuation spectra of reflected signals contained a pronounced low-frequency

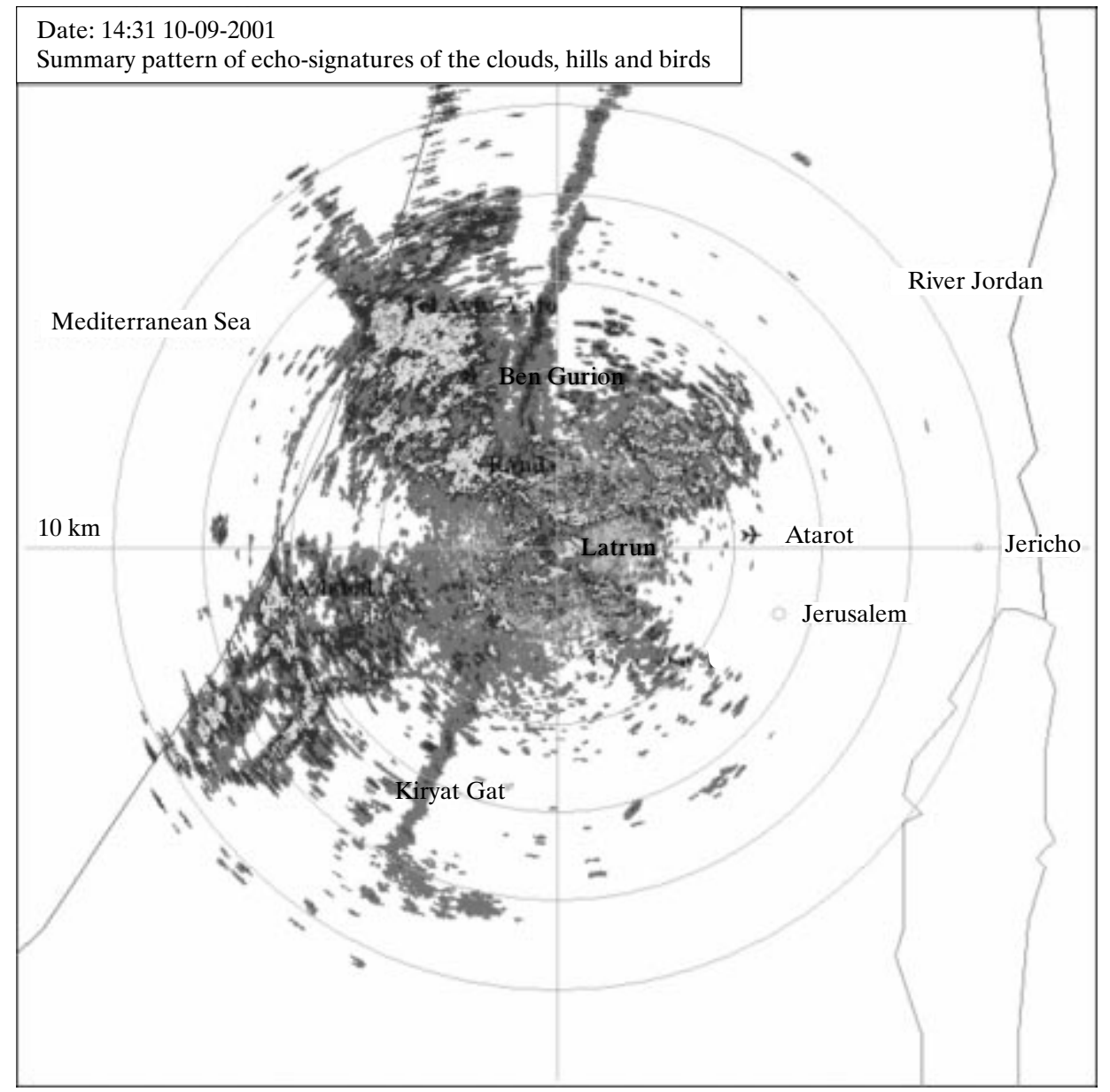

Fig. 2. Radar image of the band framing flying bird flocks, obtained by computer-produced superposition of six successive azimuth scans performed at increasing elevation. The total length of the bird flock is $100 \mathrm{~km}$.

The radar location is designated in the center of the figure, the difference between circle radiuses is $10 \mathrm{~km}$. The powerful areal radio-echo is formed by reflections from ground clutters. 
constituent, typically present in bird signals. At different levels, separate segments of the band including single radio echo points moved toward the same direction. The band was elongated toward the direction of birds' autumn migration from north to south, while the direction of the wind at the same height was different.

The radar data was found to be in complete accordance with the visual observations performed during the radar session. The particular bird flock was flying within the immediate proximity to the radar during quite a long period, at the height of 200-300 $\mathrm{m}$ and was clearly seen with the naked eye.

As can be seen from the figure, the band is directed along the coastline, remaining at the average distance of $20 \mathrm{~km}$ from the sea. The reflectivity values are different within different segments of the band, while not exceeding $30 \mathrm{~dB}$, with the exception of several individual points. This finding is accounted for by the fact that large groups of birds were flying in the same direction, while the density of the bird mass varied from group to group. The values of radio echoes obtained from the ground clutter, summed-up over 9 aerial rotations, are considerably higher than $30 \mathrm{~dB}$, at some points reaching the values of $60 \mathrm{~dB}$. This highly significant difference between the summed-up values of ground clutter signals and those obtained from birds can be considered an important indicator for distinguishing between those two types of targets. Both Figure 1 and 2 bear a significant amount of information about various aspects of bird migration. It can be also seen, however, that the screen is still 'littered' with signals from different ground and atmospheric clutter.

The samples described above demonstrate that utilising MRL-5 radar and implementing the proposed radio echo processing technique provides quality bird monitoring. In order to calculate the quantitative characteristics and velocity vectors of bird migration flow, more precise signal processing techniques are required that take into account both the spatial and the frequency characteristics of bird radio echo against the noise.

\section{CHARACTERISTICS OF RADIO ECHOES REFLECTED FROM DIFFERENT TARGETS}

The effective scattering area (ESA) for the same bird can vary by factor of 10 , depending on the bird's orientation toward the line of the radar (Houghton 1964, Eastwood 1967, Bruderer and Joss 1969). Measurements of ESA for birds were performed within an echo-absorbing chamber at different angles with respect to the antenna directional pattern (Zavirucha et al. 1977). It was shown that the signal maximum falls between $65^{\circ}$ and $115^{\circ}$, this segment corresponding to bird's lateral projection $\left(0^{\circ}\right.$ corresponds to the position of the radar beam directed at bird's beak). Another cause for ESA variations is bird's wing-flapping which can cause ESA fluctuations, from a 10-fold increase to its reduction almost down to zero (in respect to the mean value). The frequency of fluctuations was found to be $1-24 \mathrm{~Hz}$ (Chernikov 1979). The value of ESA, therefore, depends on a bird's size, its orien- 
tation toward the line of the radar and the instantaneous position of its wings. Table 3 presents the mean ESA values for different bird species at the value of radar wavelength less then $10 \mathrm{~cm}$.

The main sources of reflected signals, among them false signals, in the measurement area are ground clutter, clouds and precipitation, insects, birds, aircraft, as well as invisible atmospheric inhomogeneities caused by high air temperature gradients, and artificial obstacles of different kinds. Radar signals reflected from all those objects have a large dynamic variation range, while each type of objects has its own particular fluctuation pattern.

In order to extract bird signals from the background, it is necessary to apply selection algorithms taking into account the most characteristic features of the efficient signal. One of such features is the fluctuation pattern specific for bird signals. The signal power variation, according to different authors (Chernikov 1979), may exceed $\pm 10 \mathrm{~dB}$. Another, even more significant aspect, is the fluctuation pattern specific for bird signals, which is directly related to the frequency of wing-flapping and to the change in flight direction in respect to the radar position, all causing the corresponding changes in the bird's ESA. Comparing frequency spectra of bird signals with those obtained from other objects, one can detect birds with quite high confidence. Under the assumption that a bird does not change its flight direction during the short observation period, the fluctuation value for a bird signal can be reduced to the function of its wing-flapping. This suggests that a bird's wingflapping pattern can be estimated on the basis of the fluctuation values of its signal. Since different bird species differ in their wing-flapping patterns, the fluctuation feature together with other signal parameters, e.g. ESA-value, makes it possible to classify birds by species on the basis of signals they reflect.

Figure 3 shows the characteristic spectra of normalised signals reflected from a bird, a cloud and a local clutter. As can be seen from the figure, within the low frequency area (below $50 \mathrm{~Hz}$ ) the bird spectrum is considerably stronger than the other two spectra. In order to determine spectral characteristics typical of bird signals, 20-second samples were collected of signals reflected from: (a) 170 birds of various species, (b) 120 ground sites, (c) 190 convective and stratus clouds and (d) 95 various atmospheric inhomogeneities. The data analysis made it possible to design a low-pass filter capable of detecting birds with the confidence of $80-85 \%$. The number of erroneous identifications of birds does not exceed 20\%. It has been found that signal spectra typical of bird groups have larger frequency fluctuation range than signals spectra of individual birds, which suggests a possibility of using the proposed method for determining bird size and species.

In order to obtain the spectrum of bird signals, the observation time is to be not less than 15-20 s. This means that the fluctuation parameter can be implemented only in the manual antenna control mode in order to make the "bird - not bird" decision, as well as to determine bird species. In conditions of automatic antenna mode (4-6 scans per minute), it is currently impossible to obtain signal samples of sufficient duration. 
a

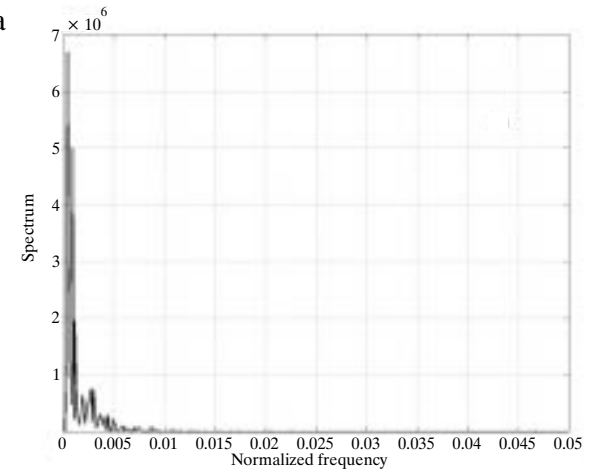

c

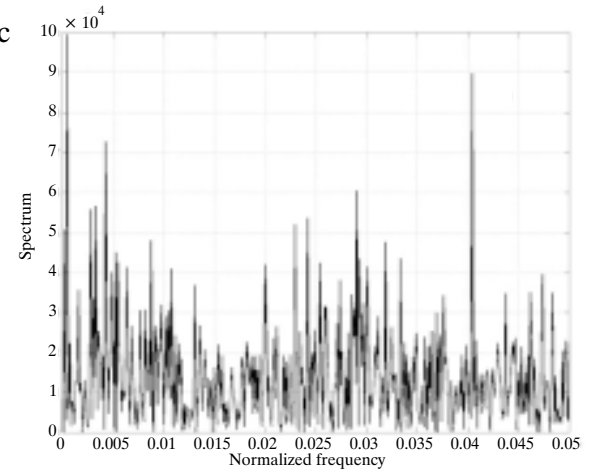

b

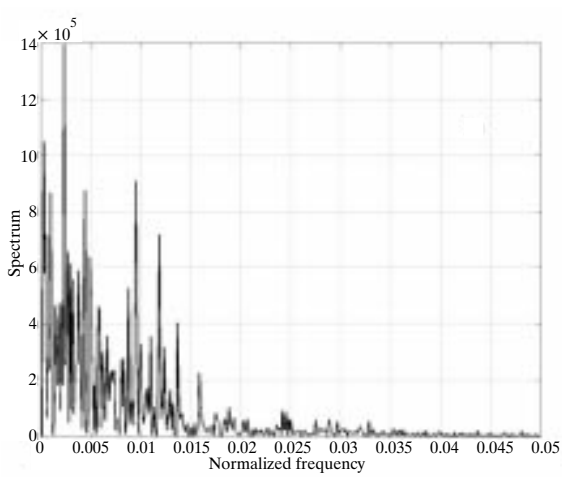

d

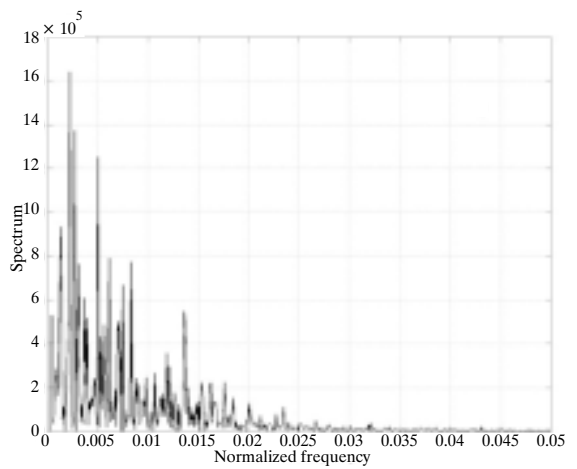

Fig. 3. Characteristic shapes of fluctuation spectra of radio-echo reflected from different targets: A. birds, B. ground clutters, C. clouds, D. atmospheric inhomogeneities.

\section{THE METHOD AND THE ALGORITHM FOR DETECTING BIRDS AND ESTIMATING THEIR FLIGHT VELOCITIES}

\section{The main idea}

The input data for the measurements is provided by radar reflectivity fields obtained by successive azimuth scans. The scans (usually 7-9 in number) are performed at fixed antenna elevation. A bird signal changes its location in space while the bird is moving. As a result of successive scans, the coordinates of the signal centre form a direct line, in case the bird does not significantly change the direction of its flight during the entire observation period. This peculiarity enables to extract the moving signal from the background of motionless objects (ground clutter, clouds, precipitation, etc.). At the same time, the information related to the signal movement can be used to estimate the velocity vector for each individual bird.

Figure 4 shows the radar reflectivity field resulting from summing-up the signals obtained in nine successive azimuth scans (this field is shown within the framed $A$-square in Figure 1). As can be seen, the field has a linear structure where each 

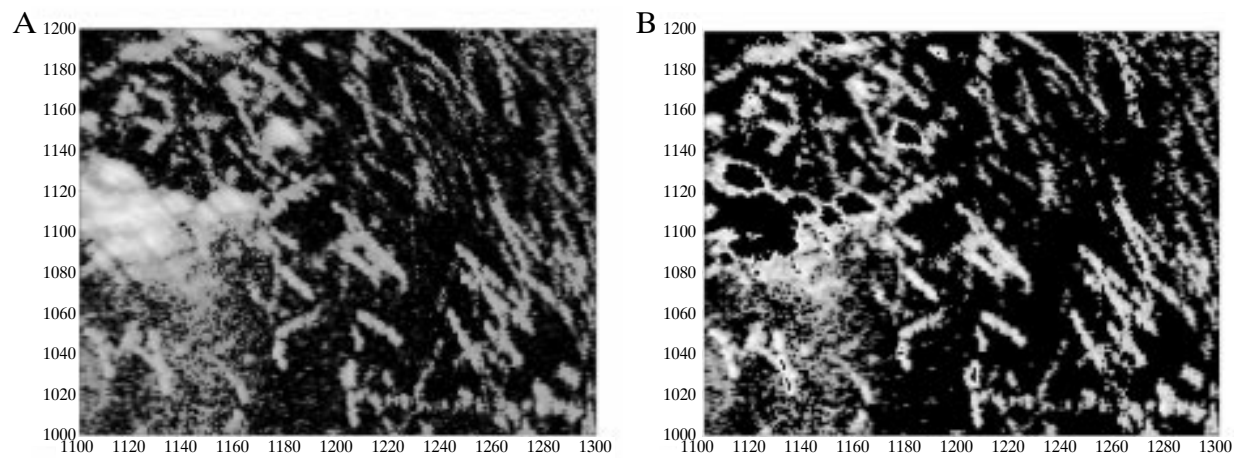

Fig. 4. Enlarged rectangular $A$ framed in Figure 1 as a target radar-scanning zone. The horizontal and the vertical scales represent distances in relative numbers. A. The total image comprising radio-echo from all reflecting objects under consideration; B. same as in Figure 4A, after signal filtering.

line corresponds to an individual bird or a bird group. The thickness of a line is determined by the radar's azimuth resolution and distance resolution (see Table 2), as well as by the recording system parameters. The lengths of the segments are proportionate to average bird velocities, while the directions of the lines correspond to the average bird flight directions. In some cases, it is possible to determine a bird species on the basis of the mean signal power with the help of Table 3 .

Table 3

Approximate radar important parameters of a bird's body for some bird species

\begin{tabular}{|l|c|c|c|c|c|}
\hline \multicolumn{1}{|c|}{ Species } & $\begin{array}{c}\text { Length of bird's } \\
\text { body }(\mathrm{cm})\end{array}$ & $\begin{array}{c}\text { Width of bird's } \\
\text { body }(\mathrm{cm})\end{array}$ & $\begin{array}{c}\mathrm{ESA}^{*} \\
\left(\mathrm{~cm}^{2}\right)\end{array}$ & $\begin{array}{c}\mathrm{CSA}^{* *} \\
\left(\mathrm{~cm}^{2}\right)\end{array}$ & $\begin{array}{c}\text { Distance } \\
\text { of detection }\end{array}$ \\
\hline Albatross & 30 & 12 & 400 & 350 & $90 \mathrm{~km}$ \\
\hline Seagull & 15 & 8 & 120 & 85 & \\
\hline Pigeon & 10 & 6 & 60 & 30 & \\
\hline Skylark & 8 & 4 & 30 & 13 & \\
\hline Sparrow & 5 & 3 & 15 & 10 & $8 \mathrm{~km}$ \\
\hline
\end{tabular}

Effective scattering area

** Approximate cross-section of flying bird

As can be seen in Figure 4A, the reflectivity field looks quite chaotic, as the lines intersect and adjoin, the signal fluctuations being considerable. The figure also shows signals reflected from other objects, as well as noises covering all the area under consideration. This indicates the complexity of the bird identification task and the need for taking into account other signal features.

\section{The algorithm}

Figure 5 shows the flowchart of the algorithm for bird identification and estimation of flight velocities. The algorithm includes three main stages: 


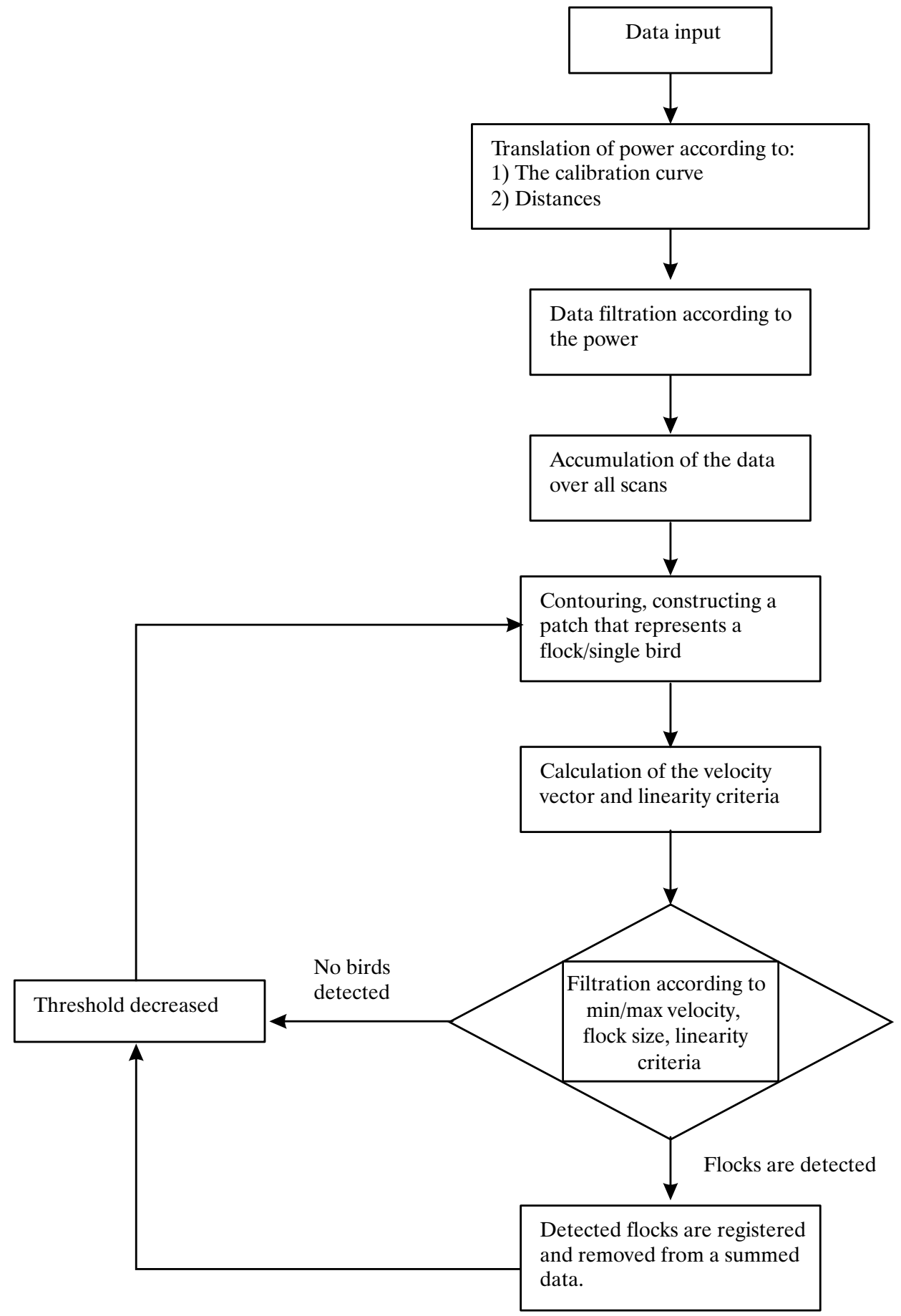

Fig. 5. The flow chart of the algorithm for selecting radio-echo from birds and estimation of their velocity vectors. 
1. Analysis of signal power and selecting signal by power value,

2. Contouring the areas occupied by a signal reflected from a bird (bird group) and selecting signals related to birds on the basis of signal power,

3. Estimating the velocity vector for each bird (bird group) and selecting bird signals by the correlation coefficient criterion.

Stages (2) and (3) are repeated at different contouring thresholds. This procedure enables to achieve equal reliability in identifying birds both within areas with rare signals (few signal-reflecting objects) and within densely covered areas (a large number of signal-reflecting objects).

\section{Signal power analysis}

According to the data obtained, at night the power of the majority of bird echoes within the distance of $40 \mathrm{~km}$ from the radar does not exceed $20 \mathrm{~dB}$, while in the daytime within the radius up to $100 \mathrm{~km}$ this value reaches $30 \mathrm{~dB}$. The analysis of signal power includes:

- calculation of signal power in accordance with the calibration curve.

- calculation of $R^{2}$ correction. At this stage the signal is de-logarithmed and its power is corrected by the following formula:

$$
S(R)=S^{*}(R) \times\left(R / R_{0}\right)^{2}-S_{N}
$$

where:

$S(R)$ - signal power to be calculated,

$S^{*}(R)$ - the power of signal received at the distance of $R$ from the radar,

$R_{0}-10 \mathrm{~km}$,

$S_{N}$ - the mean value of the noise radar power $(5 \mathrm{~dB})$.

This formula takes into account the decrease in signal power that takes place when the distance to a bird increases, as well as the fact that the power of the radar noise does not depend on this distance.

- signal restriction in accordance with the thresholds. It is assumed that values below $5 \mathrm{~dB}$ represent the radar noise, while signals above $20 \mathrm{~dB}$ (for night time) and $30 \mathrm{~dB}$ (for day time) are considered to be reflections from unrelated objects (ground clutter, etc.).

Figure 4B shows the reflectivity field summed-up after signal power selection was performed for the same area as in Figure 4A. Comparison of these two pictures shows that, as a result of the restriction performed on signal power, the areas occupied by birds can bee seen far more distinctly and there is no loss of signals obtained from individual birds (bird groups). At the same time, Figure 4 suggests that selection by signal power criterion is not sufficient, since part of the signals reflected from unrelated objects still remains in the picture.

\section{Contouring areas occupied by signals reflected from birds}

In order to contour the areas occupied by individual birds (bird groups) presented by streaks in Figure 4B, MATLAB procedures for image processing utilising morphological filters were used. The algorithm includes the following procedures: 
- bwselect: skeleton design at a prescribed reflectivity level. As a result of this procedure, the binary image of the summed-up signal field is obtained. Most of the areas within this image are closed ones.

- bwmorph: elimination of separately located points and unclosed lines within the designed skeleton. As a result of this procedure, only closed areas remain within the binary image. After signal selection, each of these areas corresponds to an individual bird (group of birds flying together).

- bwlabel: marking points within the contoured areas on the initial map, numbering the areas and calculating their quantities.

The type and the quantity of isolated areas depend significantly on the prescribed reflectivity threshold. When the threshold decreases, in spaces containing a small number of birds one observes complete separation of areas corresponding to individual birds (bird groups). At the same time, some areas occupied by a solitary bird also split into halves, thus causing the algorithm malfunction. In order to eliminate this conflict, the contouring procedure is repeated many times. At each step, i.e. algorithmic cycle, the threshold is decreased, the contours that have been identified as a solitary bird being stored and excluded from the next stage of calculations.

With the purpose of optimising the algorithm's performance, an additional procedure of spatial filtering was carried out. Figure 6 shows the layout of the contoured areas corresponding to individual birds (bird groups) within different algorithmic cycles: the $1^{\text {st }}$ cycle with 7 areas separated (A), the $15^{\text {th }}$ cycle with 25 areas separated (B) and the $30^{\text {th }}$ cycle with 64 areas separated (C).

In order to decide if the contoured area is bird-related, two criteria were used: the size of the area and the velocity at which the signal moves along the direct line within the area (the latter criterion is considered in greater detail in the next section). The minimum area occupied by a signal depends on the distance between this area and the radar and is related to resolution of the recording system (see Table 2). This area can be calculated by the formula:

where:

$$
J_{\min }=R \times \Delta R \times \Delta \phi
$$

$R$ - the distance to the radar,

$\Delta R$ - resolution value for distance,

$\Delta \phi-$ resolution value for azimuth.

The maximum area occupied by a bird is determined by the expected maximal velocity of its movement, as well as by its distance from the radar:

where:

$$
J_{\max }=(R \times \Delta \phi+\Delta R) \times V_{\max } \times \Delta T \times N
$$

$V_{\max }$ - the maximum velocity of a flying bird,

$\Delta T$ - the period of one antenna rotation,

$N-$ the number of scans.

A given area is identified as a bird echo in case its square is within the range: $J_{\min }<J<J_{\text {max }}$ Therefore, as the result of the algorithm application we obtain contoured and marked areas that correspond to an individual bird (a bird group). 


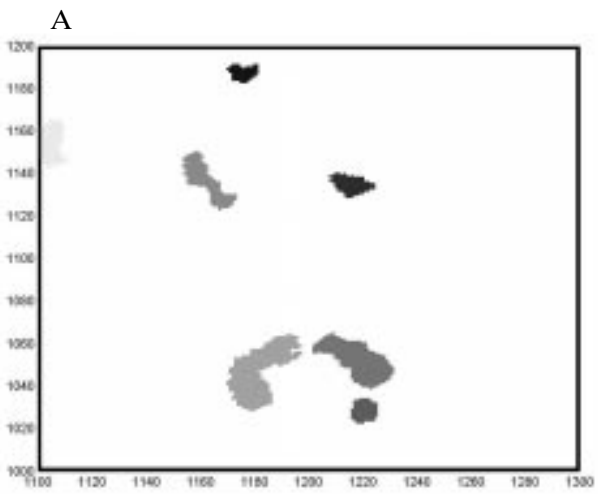

B
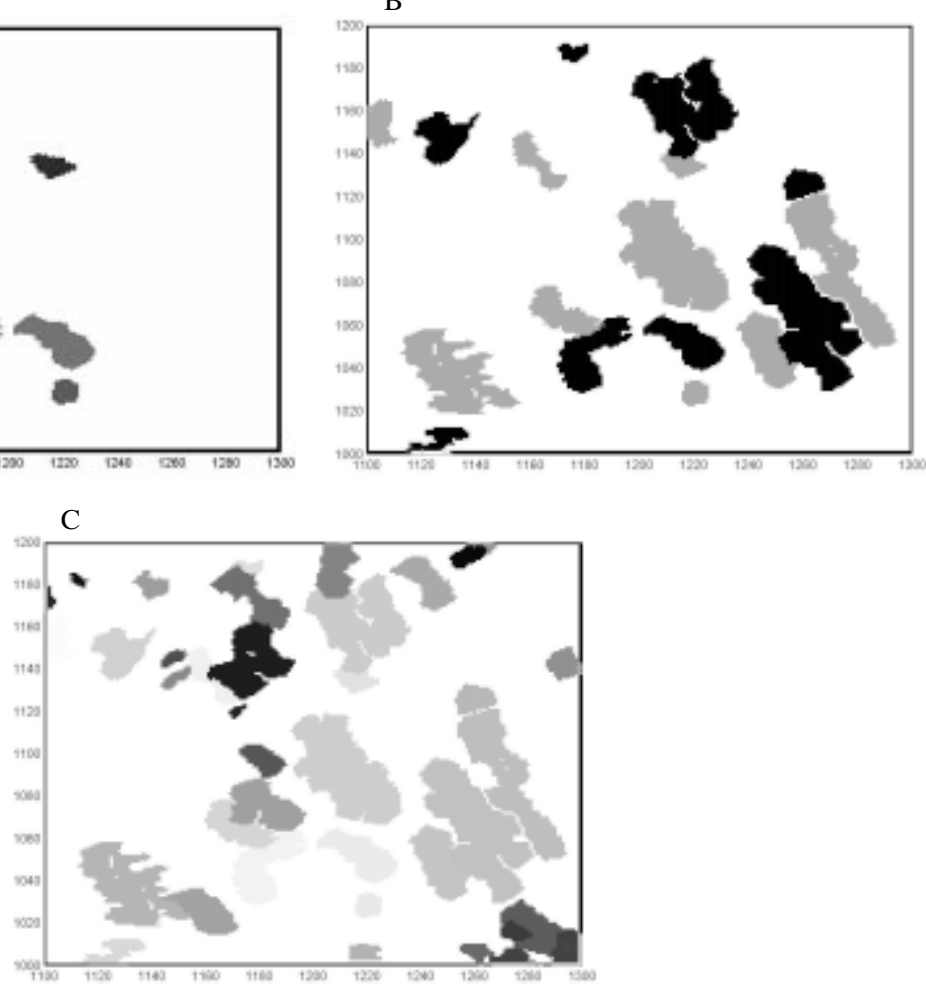

Fig. 6. Areas within $A$-radar scanning zone, representing individual birds (bird groups) contoured at different algorithm performance cycles: A. $1^{\text {st }}$ cycle ( 7 areas contoured), B. $15^{\text {th }}$ cycle $(25$ areas contoured), C. $30^{\text {th }}$ cycle (64 areas contoured).

\section{Estimation of the velocity vector}

At this stage, for each contoured area and each scan the centre coordinates $(\bar{X}, \bar{Y})$ of the signal are calculated, taking into account the signal power:

$$
\bar{X}(j)=\sum_{i} S_{i j} \times X_{i} / \sum_{i} S_{i j} \quad \bar{Y}(j)=\sum_{i} S_{i j} \times Y_{i} / \sum_{i} S_{i j}
$$

where:

$S_{i j}$ - the values of signal power,

$i-$ the number of a point within each scanning,

$j-$ the number of the scan.

Figure 7 shows the time dependencies of signal gravity centre coordinates (A and B) and the dependence of $\bar{Y}$ coordinate upon $\bar{X}$ coordinate (C). As can be seen, the points fall approximately onto a direct line, which means the bird is flying along a direct line as well.

On the basis of $\bar{X}(j), \bar{Y}(j), t(j)$ values (points of time), the mean-square linear regression dependencies $X(t), Y(t), Y(X)$ are calculated. The tangents of the slope angles for the obtained dependencies $X(t)$ and $Y(t)$ are the estimation of the bird's ve- 

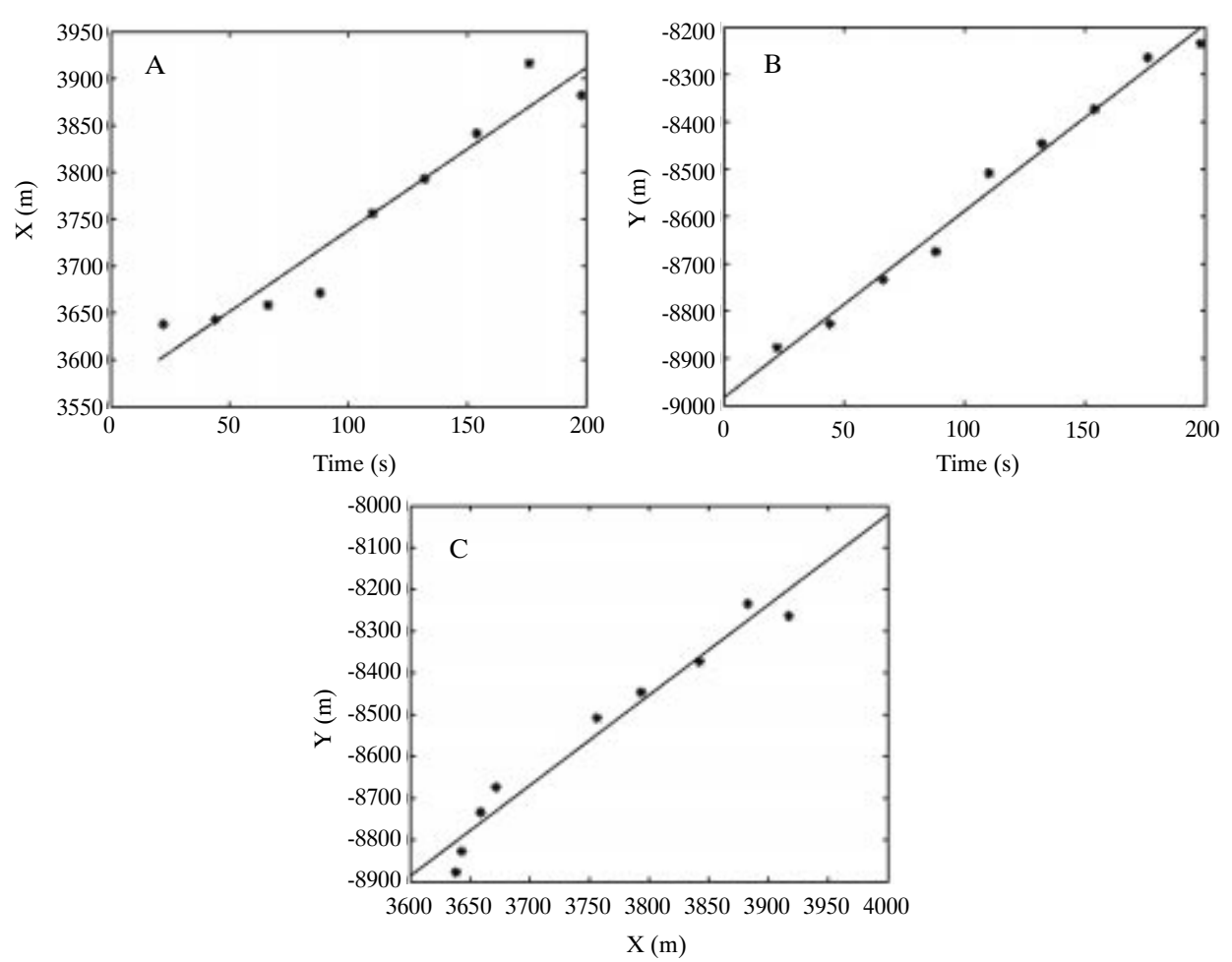

Fig. 7. The dependencies of coordinates of radio-echo center location (experimentally obtained dependencies are marked with asterisks; regression lines are designated by firm lines); A. and B. time dependencies of signal center location $-X(t), Y(t)$; C. dependence of $\bar{Y}$ coordinate on $\bar{X}$ coordinate.

locity components $V_{x}$ and $V_{y}$. Correlation coefficients $R_{x x}, R_{y y}, R_{x y}$ obtained as a result of calculating the regression dependencies, are confidence measures used in estimation of velocity components. A given signal area is identified as a bird reflection, and the calculated velocity values are accepted as relevant in case the absolute value of all the three correlation coefficients exceeds 0.75 . Otherwise, the results are not considered relevant, and the area points are returned for calculations to be performed within the next contouring cycle at an altered threshold value. The absolute value of bird's velocity is used as a supplementary criterion in case it does not exceed the prescribed value $(90 \mathrm{~km} / \mathrm{h})$.

\section{Optimization of parameters and algorithm testing}

The algorithm developed in this study comprises a large number of parameters whose values have an influence upon the efficiency of the algorithm's overall performance, the major parameters being:

- the maximum value and the power of signal identified as a bird echo,

- the range and the number of thresholds applied within the contouring procedure,

- the value of maximum correlation coefficients used as the information confidence criteria, 
- the maximum and the minimum squares occupied by a bird (bird group) echo.

As it is always the case in identification applications, prescribing "stricter" parameter values leads to a more reliable isolation of a smaller number of birds, while "milder" restrictions result in an increase of the number of birds identified, accompanied by a decrease in information reliability. To optimise the algorithm, we used a vast corpus of observation data that had been processed at different values of the parameters. Among the input data there was the a priori information available on the birds and the ground clutter, such as: (a) ESA value (see Table 3) recalculated into signal power values; (b) the mean velocities of the flying birds; (c) the direction of bird migration typical of the observation period, etc. The visual analysis was carried out with the help of various technical means, such as photographing the radar screen at different exposure values and visual comparison of data obtained after each scan.

Visual comparison of spatial maps presenting the summed-up signal to signal maps obtained at individual scans enables to identify birds with a high degree of confidence, the only exception being cases of extremely high bird concentrations over small areas. The optimum parameters for measurements and data processing have been determined, some of them shown in Table 4.

Table 4

Some optimum parameters for measurements and data processing

\begin{tabular}{|l|c|}
\hline \multicolumn{1}{|c|}{ Scan } & $15 \mathrm{sec}$ \\
\hline The number of scans at fixed elevation value & 11 \\
\hline The maximum and the minimum signal levels (summed-up over 11 scans) & $7-60 \mathrm{~dB}$ \\
\hline The number of thresholds performed in course of contouring & 30 \\
\hline The threshold correlation coefficient & 0.75 \\
\hline The minimum and the maximum velocity values & $2.5-90 \mathrm{~km} / \mathrm{h}$ \\
\hline
\end{tabular}

The algorithm and the method were tested on the basis of the data obtained within 11 scans performed on 13 May 2001 during the morning hours. Possible interference of insects causing the signal level of about 1-2 dB, was eliminated by signal restriction from below. The complete field of bird velocities obtained as a result of calculations within this measurement series is shown in Figure 8. Figure 8A shows the summed-up radio-echo at antenna elevation of $0.5^{\circ}$, formed by all the three types of reflecting objects outlined above, within the radius of $60 \mathrm{~km}$. In the figure, bird radio echoes are represented by long streaks and dots. Figure $8 \mathrm{~B}$ shows the vectors whose length corresponds to the flight velocity of detected birds. Simultaneously with the measurements, "reference" data on the birds was being obtained by photographing. As the result of the algorithm operation, 315 birds were detected with high degree of confidence, their flight velocities were estimated and found to fall within the range of $2.72-51.76 \mathrm{~km} / \mathrm{h}$. According to the visual observations, the 

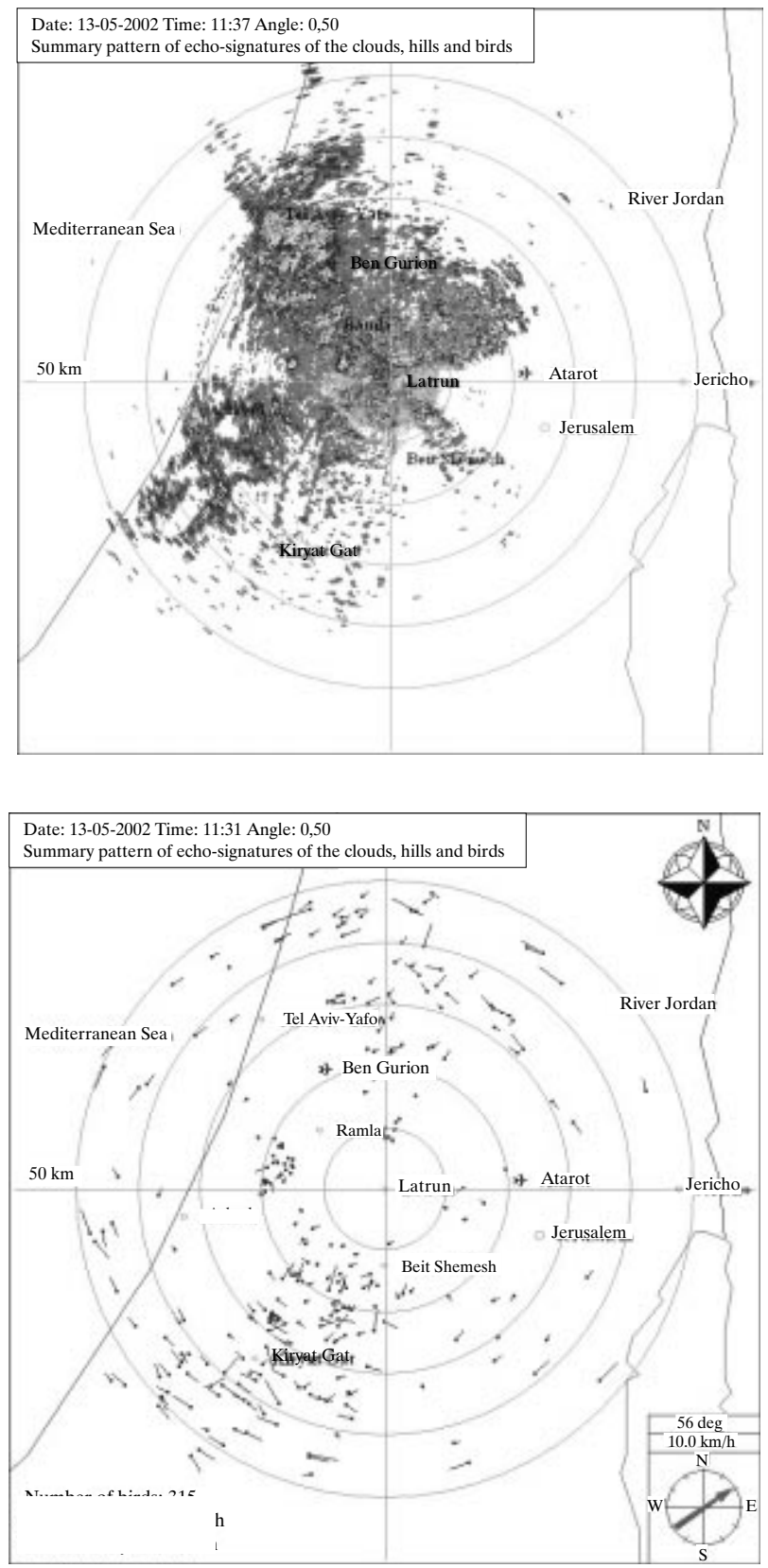

Fig. 8. Illustration of the method for selecting bird radio-echo and estimation the velocity vectors of flying birds: A. total radio-echo obtained by computer-produced superposition of eleven successive azimuth scans performed at fixed elevation value. The location of the radar is designated in the center of the chart. Radio-echoes in the form of streaks and dots are identified as reflections from birds, according to radar screen photographs analysis; B. same as A., after filtering bird echoes and estimating the vectors. A vector direction corresponds to the direction of an individual bird (bird groups) flight; vector length corresponds to the fight velocity. According to the radar screen photographs analysis, radio-echo in a vector form is identified as reflected from birds. 
actual number of birds on the radar screen during the observation period was as large as 345 . Hence, the error for this series of measurements was 30 birds. The estimated vectors (Fig. 8B) well coincide with the locations of lines and dots of the bird radio-echoes (Fig. 8A).

By comparing the figures with the photographs, it can be concluded that the algorithm developed in the present study enables to detect flying birds with high degree of confidence.

\section{CONCLUSIONS}

The algorithm that has been developed enables to identify bird signals against the background of other signal-reflecting objects and to estimate the velocity vectors of flying birds. The preliminary analysis of the algorithm's performance has demonstrated that the discovered properties of bird radio echoes and the proposed methods for signal processing make it possible to achieve high confidence in distinguishing and estimating vector fields of flying birds.

The algorithm and the designed matching software can be used as the foundation in developing a real-time program for a network of MRL-5 radars located over a vast territory. The program will allow to monitor intercontinental migrations of large bird masses, as well as to carry out studies of individual bird behaviour in course of the migration process.

In order to achieve more precise filtration of bird echoes against the background of other signals, it is proposed to supplement the set of signal properties detailed in the study with characteristics related to polarisation and fluctuation aspects of radio-echo.

\section{ACKNOWLEDGEMENTS}

The authors are especially grateful to the Ministry of Defence (MAF'AT) and the Ministry of Immigration Absorption of the State of Israel for providing financial support of the study. The authors express their gratitude to A. Kapitannikov, V. Garanin, O. Sikora for designing the software tools and performing the technical maintenance of the radar system.

\section{REFERENCES}

Abshayev M., Burtsev I., Vaksenburg S., Shevela G. 1980. Guide for use of the MRL-4, MRL-5 and MRL-6 radars in urban protection systems. Hydrometeoizdat, Leningrad.

Abshayev M., Kaplan L., Kapitannikov A. 1984. [Form reflection of meteorological targets at the primary processing of the meteorological radar signal]. Transactions of VGI 55. (in Russian)

Alfiya H. 1995. Surveillance Radar Data on Nocturnal Bird Migration over Israel, 1989-1993. Isr. J. Zool. 41: 517-522.

Bellrose F. 1967. Establishing certain parameters of hazards to aircraft by migration birds in the Missipi Flyway. Rep. for US Dep. Inter., Sport Fish. Wildl. Div. Wash. p. 74. 
Bruderer B., Joss A. 1969. Zur Registrierung and Interpretation von Echosingnaturen an enema $35 \mathrm{~cm}$ Zielvervolgungstradar. Ornithol. Beob. 66: 70-88.

Buurma L. 1999. The Royal Netherlands Air Force: Two Decades Of Bird Strike Prevention "En Route". Int. Semin. on Birds and Flight Safety in the Middle East, Israel, April, 25-26, 1999: pp. 71-83.

Buurma L., Bruderer B. 1990. The application of Radar for bird strike prevention. Bird Strike Committee Europe, the Hague: 75 pp.

Chernikov A. 1979. Radar clear air echoes. Hydrometeoizdat, Leningrad: pp. 3-40.

Dinevich L., Kapitalchuk I., Schupjatsky A. 1990. [Measurement of the microphisical characteristics of clouds and precipitation with a dual-polarization radar]. In: [Artificial modification of atmospheric processes in Moldova]. vol. 2. Kiscinev. (in Russian)

Dinevich L., Kapitalchuk I., Schupjatsky A. 1994. Use of the polarization selection of radar signals for remote sounding of clouds and precipitation. $34^{\text {th }}$ Isr. A. Conf. Aerospace Sci.: pp. 273-277.

Dinevich L., Kaplan L. 2000. On radar observation of birds migration. J. Sci. Isr. -Technological Advantages 2: 89-93.

Dinevich L., Leshem I., Gal A., Garanin V., Kapitannikov A. 2000. Study of birds migration by means of the MRL-5 radar. J. Sci. Isr. -Technological Advantages 2: 94-104.

Dinevich L., Leshem I., Sikora O. 2001. Radar Observation Analysis of Season Bird Migration in Israel at Night. J. Sci. Isr. - Technological Advantages 3: 77-101.

Eastwood E. 1967. Radar ornithology. Methuen, London: p. 278.

Ganja I., Zubkov M., Kotjazi M. 1991. [Radar ornithology]. Stiinza: pp. 123-145. (in Russian)

Gauthreaux S.A. 1970. Weather radar quantifications of bird migration. Bio-Sciences 20, 1: 17-20.

Gauthreaux S.A., Sidney A.Jr., Belser C.G. 1998a. Displays of Bird Movements on the WSR-88D: Patterns and Quantification. Weather and Forecasting 13: 453-464.

Gauthreaux S.A., Sidney A.Jr., Mizrahi D.S., Belser C.G. 1998b. Bird Migration and Bias of WSR-88D Wind Estimates. Weather and Forecasting 13: 465-481.

Glover K., Hardy K., Landry C. 1966. Radar characteristics of known insects in free flight. Proc. $12^{\text {th }}$ Weather Radar Conf. Amer. Met. Soc., Boston: pp. 254-258.

Hajovsky R., Deam A., La Grone A. 1966. Radar reflections from insects in the lower atmosphere. IEEE Trans. on Antennas and Propagation 14: 224-227.

Houghton E. 1964. Detection, recognition and identification of birds on radar. World Conf. Radio Met., Amer. Met. Soc., Boston: pp. 14-21.

Leshem Y. 1999. Developing a Real Time Warning System in the Middle East; from Vision to Reality. Int. Semin. on Birds and Flight Safety in the Middle East, Israel, April, 25-26, 1999: pp. 45-69.

Richardson R., Stacey J., Kohler H., Naka F. 1958. Radar observation of birds. Proc. $7^{\text {th }}$ Weather Radar Conf., Am. Met. Soc., Boston: pp. D1-D8.

Rinehaart R. 1966. Radar detection of birds in New Mexico. Preprint, Illinois State Water Survey: p. 6.

Zavirucha V., Saricev V., Stepanenko V, Shepkin U. 1977. Study of the dispersion characteristics of the meteorological and ornitological objects in echo-free cameras. Proc. Main Geophysic Observatory, \#395: pp. $40-45$.

Zrnic D.S., Ryzhkov A.V. 1998. Observations of insects and birds with a polarimetric radar. IEEE Trans. on Geoscience and Remote Sensing 36, 2: 661-668. 